\title{
AVALIAÇÃo DA DISPONIBILIDADE DE EQUIPAMENTO DE DIAGNÓSTICO POR IMAGEM EM USO NA CIDADE DE CERES - GO
}

EVALUATION OF THE AVAILABILITY OF DIAGNOSTIC EQUIPMENT BY IMAGE IN USE IN THE CITY OF CERES - GO

\section{Mateus Ferreira da Silva}

Discente do Curso Superior de Tecnologia em Radiologia, Faculdade Evangélica de Ceres, Ceres-GO

E-mail: mateus85ferreira@gmail.com

\section{Wisley Alleff Santana de Carvalho}

Discente do Curso Superior de Tecnologia em Radiologia, Faculdade Evangélica de Ceres, Ceres-GO

E-mail: wisleykcs0505@gmail.com

\section{Carla Danielle Dias Costa}

Docente do Curso Superior de Tecnologia em Radiologia, Faculdade Evangélica de Ceres, Ceres-GO

E-mail: carladaniellebm@gmail.com

\section{RESUMO}

INTRODUÇÃO: O Brasil possui um sistema público de saúde cujo princípio básico é garantir, a toda população, acesso aos diversos serviços por ele oferecidos. Desta maneira, foi criado pela Constituição Brasileira o Sistema Único de Saúde (SUS), que oferece gratuitamente acesso a diversos serviços, entre esses os exames de diagnósticos por imagem. OBJETIVOS: $O$ estudo verifica a distribuição dos equipamentos de diagnóstico por imagem, Equipamentos como: tomografos computadorizados, ressonância magnética, radiografia convencional e mamografias, disponíveis na cidade de Ceres - GO. METODOLOGIA: Trata-se de uma pesquisa descritiva por levantamento de dados no Cadastro Nacional de Estabelecimentos de Saúde (CNES), disponibilizados no sistema DATASUS, feito sobre clinicas públicas e privadas. O período de busca dos dados foi em novembro de 2018, a respeito do número de equipamentos de diagnóstico por imagem, disponíveis na cidade de Ceres - GO. RESULTADOS E DISCUSSÃO: Com base nas informações do Cadastro Nacional de Estabelecimentos de Saúde, disponibilizados no sistema DATASUS, foi possível identificar que a cidade de Ceres possui 34 clínicas, possuindo um total de 124 equipamentos de diagnóstico por imagem, destes estão em uso 117 aparelhos dos quais realizam exames complementares de diagnóstico por imagem. CONCLUSÃO: Embora o processo de cadastramento de informações no banco de dados do CNES/ DATASUS ainda não tenha alcançado sua última etapa, a certificação, os dados disponíveis relativos ao município de Ceres -GO, já permitem uma análise bastante realista do equipamentos de diagnóstico por imagem instalado na cidade, ainda que a maior parte das clínicas atendem somente o público privado, mesmo que a saúde seja um direito de todos os cidadãos.

Palavras-chave: Exames de imagem, Equipamentos para diagnóstico, SUS. 


\begin{abstract}
INTRODUCTION: Brazil has a public health system whose basic principle is to guarantee the entire population access to the various services offered by it. Thus, the Brazilian Constitution created the Unified Health System (SUS), which offers free access to various services, including diagnostic imaging exams. OBJECTIVES: The study verifies the distribution of diagnostic imaging equipment, such as computed tomography, magnetic resonance imaging, conventional radiography and mammograms, available in the city of Ceres - GO. METHODOLOGY: This is a descriptive survey by data collection in the National Register of Health Facilities (CNES), available in the DATASUS system, made on public and private clinics. The data search period was in November 2018, regarding the number of diagnostic imaging equipment available in the city of Ceres - GO. RESULTS AND DISCUSSION: Based on information from the National Register of Health Facilities, available in the DATASUS system, it was possible to identify that the city of Ceres has 34 clinics, with a total of 124 diagnostic imaging equipment, of which 117 devices are in use. of which perform diagnostic imaging exams. CONCLUSION: Although the process of registering information in the CNES / DATASUS database has not yet reached its last stage, the certification, the available data for the municipality of Ceres - GO, already allow a very realistic analysis of diagnostic equipment by image installed in the city, although most clinics serve only the private public, even if health is a right of all citizens..
\end{abstract}

Keywords: Imaging, Diagnostic Equipment, Unified Health System of Brazil (SUS). 


\section{INTRODUÇÃO}

Desde 1895, quando Wilhelm Conrad Röntgen descobriu o raio X e revolucionou o meio científico, novas tecnologias passaram a existir e, atualmente, a radiologia presencia uma constante mudança, especialmente em função dos progressos na área dos exames diagnósticos.

A informatização trouxe uma série de benefícios para diversos setores da sociedade contemporânea, especialmente para as áreas da saúde. A aplicação dos benefícios e suas relações com os distintos setores da saúde, promoveram um importante salto de qualidade e de economia de tempo na clínica diária, na terapêutica e, principalmente, expressivo auxílio na obtenção dos diagnósticos. As imagens interpretadas pelas radiografias, atravessaram quase um século e novas tecnologias abriram os horizontes para os exames de diagnósticos (SILVA, 2011).

Os exames de diagnósticos colaboram para o desenvolvimento da assistência à saúde e auxiliam na tomada de decisão do profissional médico, sejam eles de rotina ou de rastreamento, determinando uma diagnose mais precisa do paciente. Os exames de diagnóstico são divididos em clínicos, laboratoriais e de imagem, de modo que os mesmos permitem avaliação completa do paciente. Logo, cada exame possui sua peculiaridade (SOUZA, 2014).

Os exames clínicos realizam a coleta de sinais e sintomas para que se possam elaborar hipóteses de diagnóstico. Deve-se cumprir uma sequência lógica, completa e minuciosa em duas fases: anamnese e exame físico. Os exames laboratoriais são uma série de exames indicados, afim de diagnosticar ou atestar uma doença, os exames mais comuns são os de sangue, conhecido como hemograma e os de fezes, urina. Além desses, existem uma série de exames laboratoriais para diferentes tipos de situações. Os exames laboratoriais têm por objetivos prover resultados claros e precisos, proporcionando, assim, ao profissional médico informações e indicadores favoráveis e capazes de constituir diagnósticos, corretos e apurados dos exames (BONETTI, 2016; ECHER, 2013).

Já o exame de imagem permite ver o interior do corpo, utilizando diferentes formas de energia, como raios $\mathrm{X}$, ultrassonografia, ressonância magnética, os quais são de suma importância para avaliação da estrutura e funcionamento adequado do organismo. Além de serem completamente seguros, são a melhor escolha para diagnósticos mais precisos, podendo ser feito de diferentes formas. Com os exames é provável avaliar a região onde está a doença, 
assim como a sua extensão, auxiliando na decisão de qual será a melhor forma para conduzir o tratamento. Quando os exames estão atualizados, as chances de diagnósticos precoce aumentam, e consequentemente as chances de cura, colaborando assim, para o sucesso do tratamento. Tornando-se grandes aliados da medicina preventiva no diagnóstico e investigação de doenças (SOUZA et al, 2014; MAGNUS, 2016).

Portanto, os exames de imagem podem utilizar-se da radiação ionizante e não ionizante. A radiação ionizante é caracterizada como ondas de grande poder energético capazes de extrair elétrons das camadas orbitais do átomo, isso acontece devido a um fenômeno chamado de ionização, por isso, deve ser usada de maneira racional, sendo preciso analisar o risco relacionado aos benefícios. Tem-se como exemplo os raio-x, raios gama, partículas alfa e betas. Já a radiação não ionizante é aquela que não possui carga elétrica suficiente para ionizar átomos ou moléculas, tendo como exemplo a frequência ultravioleta, luz visível, infravermelho (MADRIGANO et al, 2014; OKUNO, 2013).

$\mathrm{O}$ exame de Raios- $\mathrm{X}$ foi o primeiro exame radiológico a ser coligado na área médica, no entanto com o proceder dos anos novos exames e equipamentos foram sendo desenvolvidos e cada vez mais sofisticados, tornando-se mais precisos e determinados, além de serem mais solicitados na prática médica contemporânea. A partir da simples visualização do esqueleto humano por meio dos Raios-X, a radiologia tem-se evolucionado a ponto de atualmente não se limitar a simples análise de composições anatômicas, com capacidade de acompanhar modificações funcionais (medicina nuclear) ou até mesmo concretizar associações de técnicas, possibilitando uma avaliação muito mais rica e completa. Os exames de imagem mais utilizados nos dias de hoje são a tomografia computadorizada, ressonância magnética, radiologia convencional, densitometria óssea, mamografia, ecografia tridimensional e ultrassonografia (CARVALHO, 2017; NASCIMENTO et al, 2015).

A população brasileira tem buscando mais acesso aos serviços de saúde, devido a maior mobilização para prevenção de doenças. Isso faz com que os estabelecimentos de saúde sejam conduzidos a adquirir equipamentos mais modernos, seguros, com o intuito de se conseguir fornecer os serviços de diagnóstico por imagem de qualidade e mais eficiente durante o processo saúde/doença (CAMPANA, 2011).

Assim, a partir do exposto esse trabalho teve como objetivo verificar a distribuição dos equipamentos de diagnóstico por imagem como: tomografia computadorizada, ressonância magnética, radiografia convencional e mamografias, disponíveis na cidade de Ceres - GO. 


\section{METODOLOGIA}

Trata-se de uma pesquisa descritiva por levantamento de dados no Cadastro Nacional de Estabelecimentos de Saúde (CNES), disponibilizados no sistema de informática do sistema único de saúde (DATASUS), a respeito do número de equipamentos de diagnóstico por imagem, disponíveis na cidade de Ceres - GO.

Foram considerados estabelecimentos de saúde públicos e privados que realizam exames de diagnóstico por imagem e que atendem a população de forma particular ou por convênio com o Sistema Único de Saúde (SUS), o período de busca dos dados foi em novembro de 2018 através do departamento de informática do mesmo, o DATASUS.

A coleta de dados foram levantadas as quantidades existentes de: tomógrafo computadorizado, ressonância magnética, Ultrassom (convencional, doppler colorido e Ecógrafo), mamógrafo (comando simples e com Estereotáxia) e aparelho de raios X dentária e ósseo, considerando raios $\mathrm{X}$ até $100 \mathrm{~mA}$, raios $\mathrm{X}$ de 100 a $500 \mathrm{~mA}$, raios $\mathrm{X}$ com mais de 500 $\mathrm{mA}$, raios $\mathrm{X}$ com fluoroscopia, raios $\mathrm{X}$ para densitometria óssea $\mathrm{e}$ raios $\mathrm{X}$ para hemodinâmica.

O número de equipamentos de imagem em uso, por cem mil habitantes, de categorias selecionadas em um determinado espaço geográfico, do ano considerado, foi calculado, com intuito de analisar variações geográficas e temporais da oferta da distribuição de equipamentos de imagem em saúde para a região, sendo possível identificar se há situações de desigualdade.

O método de cálculo utilizado de equipamentos em uso, por cem mil habitantes, por categoria selecionada dá-se pela seguinte equação:

Média anual do número mensal de equipamentos de imagem da categoria específica População total residente, ajustada para o meio do ano $\times 100.000$

A portaria $\mathrm{GM} \mathrm{n}^{\circ} 1.101$, de 12/06/2002, determina as quantidades mínimas necessárias para cada categoria de equipamento, sendo: Mamógrafo: 1 por 240.000 habitantes (ou 0,42 por 100.000 habitantes); o Raio-X simples: 1 por 25.000 habitantes (ou 4 por 100.000 habitantes); o Raio-X para densitometria óssea: 1 por 140.000 habitantes (ou 0,71 por 100.000 habitantes); o Raio-X odontológico: 1 por 25.000 habitantes (ou 4 por 100.000 habitantes); o 
Raio-X com fluoroscopia: não há parâmetros; o Raio-X para hemodinâmica: não há parâmetros; o Ressonância magnética: 1 por 500.000 habitantes (ou 0,2 por 100.000 habitantes); o Tomógrafo: 1 por 100.000 habitantes; o Ultrassom: 1 por 25.000 habitantes (ou 4 por 100.000 habitantes). Os valores nacionais são valores médios, que não levam em consideração as variações regionais de densidade demográfica e pode haver algumas informações desatualizadas na base de dados demográficos, principalmente em anos que não há a realização do censo.

Análise estatística de dados foi realizada por meio dos equipamentos em uso, por cem mil habitantes, por categoria foram calculados de acordo com o método de cálculo, disponibilizado pelo CNES baseado na população da região de Ceres - GO, e os demais dados foram analisados por métodos estatísticos simples (tais como distribuição de frequências, média).

Os resultados foram expressos em gráficos, tabelas de forma descritiva. Para tabulação dos dados foram utilizado o software da Microsoft@ Office Excel® 2007 (12.0.6787.5000) SP3 MSO (12.0.6785.5000) Versão 10.0.16299 compilações 16299.

\section{RESULTADOS E DISCUSSÃO}

De acordo com o Instituto Brasileiro de Geografia e Estatística (IBGE, 2010) a população residente em Ceres-GO, conforme o último censo em 2010 era de 20.722 habitantes, sendo que aproximadamente 49\% (10.144) era do sexo masculino e 51\% (10.578) do sexo feminino. Já em 2018 a população estimada é de 22.074 habitantes. O município de Ceres está localizado na Mesorregião do Centro Goiano, cerca de $170 \mathrm{~km}$ de Goiânia, ocupando uma área de 214,322 km2. Sendo limitado pelos municípios de Ipiranga de Goiás, Carmo do Rio Verde, Rialma, Santa Isabel, Rubiataba e outros (IBGE, 2018). Vê-se que a cidade de Ceres - GO, atende e alcança a vários municípios, oferecendo seus serviços de diagnóstico por imagem.

Segundo Monteiro (2015) o diagnóstico por imagem, é conhecida como exame de imagem, é uma particularidade médica que faz uso das tecnologias de imagem para efetivação de diagnósticos. Referente às técnicas e procedimentos empregados para instituir imagens do corpo humano para análise clínica. A imagiologia exerce uma função essencial no processo de cuidados de saúde na comunicação médica, educação, investigação e diagnóstico. Camilo et al (2016) asseguram que com técnicas de imagem cada vez mais aprimoradas, o diagnóstico 
por imagem, não apenas revela a composição do corpo em detalhe, como do mesmo modo a função dos tecidos dentro do corpo. Ainda que há muitos tipos de equipamento de imagem, as distintas modalidades de imagem obtidas são de forma analógica ou digital.

Bushong (2010) menciona que os exames de imagem podem utilizar-se de radiação ionizante ou não, as quais se distinguem quanto aos seus efeitos ao interagirem com a matéria, que são raios x, tomografia computadorizada, mamografia, densitometria óssea, ressonância magnética que utiliza radiação não ionizante e o ultrassom que consiste na conversão de ondas eletromagnéticas em ondas mecânicas. Em seguida, Okuno (2013), diz que a radiação é energia que se reproduz no espaço, com início a uma fonte emissora. Ela pode interatuar com o corpo humano e promover alterações as estruturas celulares. Ela se expõe em forma de partícula atômica ou subatômica energéticas tais como partículas alfa, elétrons, pósitrons, prótons, nêutrons etc. Okuno (2013), acrescenta destacando que a radiação pode mostrar-se também em forma de onda eletromagnética, composta de campo magnético oscilante e campo elétrico.

Assim, são diversos os serviços oferecidos pelo SUS e, entre eles, estão os exames de diagnóstico por imagem. O CNES, em conjunto com o DATASUS, fornece a relação de equipamentos necessários para a realização destes exames, disponíveis no sistema público de saúde. Silva e Caxias (2016) descrevem que a criação do programa SUS, tem como base atendimento igualitário as pessoas, tendo como base os seus princípios nos quais são: universalidade, integralidade, igualdade. A construção do sistema foi influenciada pela reforma sanitária brasileira, desde sua criação o SUS produz serviços em unidades de saúde incluindo hospitais públicos e privados, desta forma a venda de prestação de serviços à saúde cresce em redes pública e privadas.

Ainda Silva e Caxias (2016) embora haja prestação dos mesmos serviços de saúde entre a rede pública e privada, há uma procura maior do atendimento na rede privada, por conta dos planos de saúde. Já no atendimento do SUS se dá entre a população de baixa renda, tem-se a demora entre a marcação e a realização dos exames, diante disso a rede privada se difere, pois seu alto investimento nos aparelhos, e equipamento, visando um atendimento de qualidade devido à concorrência. Entretanto, Couto Filho (2016) afirma que todos deveriam ser atendidos da mesma forma, pois, o direito à saúde é assegurado pela Constituição Federal do Brasil, 1988 (CFB), em seu artigo $6^{\circ}$ como direito fundamental, garante que, o direito a saúde é um direito social para todos.

Com base nas informações do Cadastro Nacional de Estabelecimentos de Saúde (CNES) disponibilizados no sistema DATASUS, foi possível identificar que a cidade de 
Ceres possui 34 estabelecimentos de saúde, que realizam exames complementares de diagnóstico por imagem. Estes estabelecimentos fazem atendimento à população de modo particular, por planos de saúde, por meio de convênio e o Sistema Único de Saúde (SUS).

Andrade (2017) complementa afirmando que a CFB estabelece uma diminuição contra os preconceitos das desigualdades sociais com o objetivo de finalizar a prática justiça social e garantindo a todos o direito a um tratamento de saúde integral e totalmente gratuito. A saúde é um dos direitos humanos que possuem valor elevado. Araújo et al (2016) relatam que as informações a respeito do sistema de saúde, sua disponibilidade, distribuição geográfica, recursos tecnológicos e humanos que se encontram disponíveis e de qualidade são elementos que intervêm no acesso à saúde. Assim, ao avaliar a quantidade de equipamentos existentes na cidade de Ceres, foi identificado 34 clínicas, possuindo um total de 124 equipamentos de diagnóstico por imagem, destes 117 aparelhos estão em uso, demonstrado a seguir no Gráfico 1 .

Gráfico 1: Distribuição de equipamentos de diagnose por imagem existentes e em uso na cidade de Ceres Goiás - 2018. 


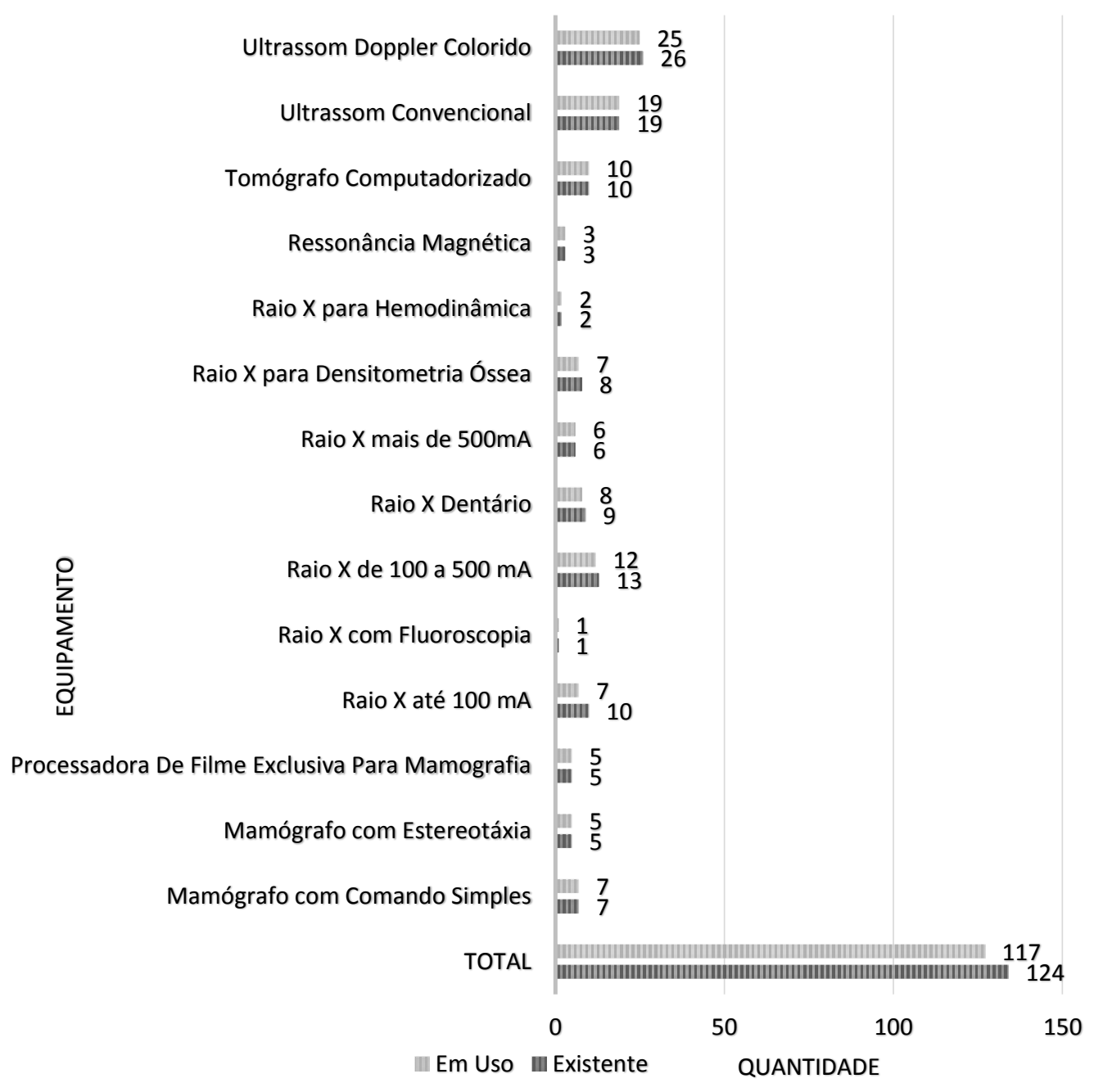

Fonte: DATASUS, 2018.

Por meio do gráfico 1 acima, percebe-se que os equipamentos existentes na cidade de Ceres GO, são capazes de atender com qualidade a todos as pessoas que precisam realizar o exame por imagem, sendo eles de rede privada ou pública. De acordo com levantamentos realizado pelo CNES a região possui vinte e seis $(20,97 \%)$ Ultrassom Doppler Colorido, Dezenove $(15,33 \%)$ Ultrassom Convencional, dez (8,07\%), Tomógrafo Computadorizado, três $(2,42 \%)$, Ressonância Magnética, dois $(1,61 \%)$ Raio X para Hemodinâmica, oito $(6,45 \%)$ Raio X para Densitometria Óssea, seis $(4,83 \%)$ Raio X mais de 500mA, nove (7,26\%) Raio X Dentário, treze $(10,49 \%)$ Raio X de 100 a 500 mA, um (0,8\%) Raio X com Fluoroscopia, dez ( 8,06\%) Raio X até $100 \mathrm{~mA}$, cinco (4,03\%) Processadora De Filme Exclusiva para mamografia, cinco (4,03\%) Mamógrafo com Estereotáxia, sete (5,65\%) Mamógrafo com comando Simples.

Logo, em 13 das 34 Clínicas de diagnóstico por imagem de Ceres são aceitos usuários do sistema único de saúde (SUS), existem exceções em que algumas dessas 13 clínicas 
atendem não somente a usuários do SUS, mas também usuários da rede privada, já as 21 outras clínicas oferecem atendimento exclusivo somente a usuários da rede privada de saúde.

Ao avaliar o índice de equipamentos em uso, com a fórmula e cálculo, percebe-se que a cidade de Ceres apresentou 124 equipamentos de diagnóstico por imagem. Quando calculados por categoria de exames, observou -se os índices de 10 tomógrafos, três aparelhos de ressonância, 17 Mamógrafos, 9 Raios-x odontológico, 1 Raios-x fluoroscopia, 2 Raios-x para hemodinâmica, 29 Raios-X simples, 55 ultrassons e oito Raios-X para densitometria óssea, ressaltando que todos os dados expressos acima abordam o número de equipamentos existentes. De acordo com a Quadro 1 pode-se fazer uma comparação de equipamentos conforme a Portaria GM n ${ }^{\circ} 1.101$, de 12/06/2002 e os dados obtidos da cidade de Ceres. No quadro 1 são expressos a média de equipamentos de diagnóstico por imagem de Ceres-go e a média nacional, ambos para cada cem mil habitantes.

Quadro 1 - Dados de acordo com a portaria GM n ${ }^{\circ} 1.101$, de 12/06/2002 que compara a média nacional por cem mil habitantes e a média da cidade de Ceres.

\begin{tabular}{|l|l|l|}
\hline EQUIPAMENTOS & PORTARIA & CERES \\
\hline Mamógrafos & 0,4 & 7,7 \\
\hline Raio-X simples & 4 & 13,1 \\
\hline Raio-X para DMO & 0,71 & 3,6 \\
\hline Raio-X odontológico & 4 & 4,1 \\
\hline Raio-X com fluoroscopia & $\begin{array}{l}\text { sem } \\
\text { parâmetros }\end{array}$ & 0,5 \\
\hline Raio-X para hemodinâmica & $\begin{array}{l}\text { sem } \\
\text { parâmetros }\end{array}$ & 0,9 \\
\hline Ressonância magnética & 0,2 & 1,4 \\
\hline Tomógrafo & 1 & 4,5 \\
\hline Ultrassom & 4 & 24,9 \\
\hline
\end{tabular}

Ao comparar os parâmetros de Ceres com os definidos pela Portaria GM n ${ }^{\circ} 1.101$, de 12/06/2002 com o número de equipamentos para cada cem mil habitantes, pode-se observar que a cidade de Ceres possui um grande número de equipamentos de diagnóstico por imagem, 
o que revela que a cidade atende as necessidades de sua população e até mesmo da população em seus entornos. No mesmo sentido, mas em nível nacional, Nascimento e Nascimento (2012), ao realizar o mapeamento dos equipamentos de saúde de um município da Paraíba, do mesmo modo chegaram à conclusão que os mesmos se concentram em pequenas áreas do município, fazendo com que boa parte da população tenha que se deslocar para ter acessos a estes serviços. Assim, como acontece na cidade de Ceres - GO, as cidades vizinhas recorrem a cidade para efetuarem seus exames de diagnósticos.

Conforme o quadro 2 acima, observa-se que dos 124 aparelhos de diagnóstico por imagem disponíveis, somente 117 encontram disponíveis no SUS para que seja utilizado pelos usuários do SUS.

Quadro 2 - Equipamentos de diagnóstico por imagem existentes e em uso disponíveis no SUS.

\begin{tabular}{|l|l|l|}
\hline Equipamentos de diagnóstico por imagem & Existente & Em Uso \\
\hline Mamógrafo com Comando Simples & 7 & 7 \\
\hline Mamógrafo com Estereotáxia & 5 & 5 \\
\hline $\begin{array}{l}\text { Processadora de Filme Exclusiva para } \\
\text { Mamografia }\end{array}$ & 5 & 5 \\
\hline Raio X até 100 mA & 10 & 7 \\
\hline Raio X com Fluoroscopia & 1 & 1 \\
\hline Raio X de 100 a 500 mA & 13 & 12 \\
\hline Raio X Dentário & 9 & 8 \\
\hline Raio X mais de 500mA & 6 & 6 \\
\hline Raio X para Densitometria Óssea & 8 & 7 \\
\hline Raio X para Hemodinâmica & 10 & 2 \\
\hline Ressonância Magnética & 3 & 3 \\
\hline Tomógrafo Computadorizado & 10 \\
\hline Ultrassom Convencional & & \\
\hline
\end{tabular}




\begin{tabular}{|l|l|l|} 
Ultrassom Doppler Colorido & 26 & 25 \\
\hline Ultrassom Ecógrafo & 10 & 10 \\
\hline TOTAL & 124 & 117 \\
\hline
\end{tabular}

Segundo Camilo et al (2016), o direito à saúde no Brasil, é um direito que solicita do Estado prestações positivas referentes a garantia, melhorias na saúde, não sendo apenas a rede privada ter atendimentos de qualidade, mas também a rede pública atender os seus pacientes de modo satisfatório e com qualidade, os quais possam contar com o mesmo número de quantidade de equipamentos oferecidos aos da rede privada, assim também assistência pública. Conforme Benevides (2014) a desigualdade é confirmada por pesquisa do Instituto de Estudos de Saúde Suplementar (IESS) onde 22 estados não tinham tomógrafos suficientes em hospitais públicos para atingir a taxa recomendada pela OMS. Desses, 15 eram das regiões Norte e Nordeste. Como por exemplo Rondônia, tem 4,7 vezes o número de mamógrafos recomendados pela OMS. E tem 5,44 vezes o número de aparelhos de ressonância. Enquanto isso, o Amapá não cumpre a recomendação e tem um aparelho para ser usado pelo SUS e pela rede privada. No entanto, esses equipamentos deveriam ser alocados para ter o uso maximizado, atendendo o maior número de pessoas nas duas redes, sendo ela privada ou pública.

Leyton et al (2014) enfatizam que a utilização da radiação ionizante para a realização de diagnósticos e terapêuticos vem se desenvolvendo anualmente, pelo motivo do desenvolvimento dos equipamentos e a promoção ao exame radiográfico. A radiação para fins diagnóstico médico ocasiona benefícios, permitindo que seja possível detectar tumores e fraturas na radiografia convencional, tomografia computadorizada, mamografia, e o tratamento de doenças que utiliza a radioterapia como no caso do câncer. A radiação do mesmo modo se encontra presente na medicina nuclear, para averiguar a fisiologia dos órgãos e dos sistemas do corpo humano.

Por conseguinte, Gasparin (2013) menciona que as radiações não ionizantes são correspondentes às que não têm energia satisfatória para ionizar os átomos e as moléculas da matéria com que agem mutualmente. Em uma ocasião, a radiação não-ionizante é constituída por fótons de energias de valor mínimo do que os da radiação ultravioleta. Assim, por causa dessa constância baixa, essa qualidade de radiação não possui capacidade de fazer com que os 
elétrons da esmagadora maioria dos átomos sejam extraídos. a seguir ao analisar os dados do Gráfico 2 foi obtido a porcentagem de equipamentos com radiações ionizantes e radiações não ionizantes da cidade de Ceres-GO.

Gráfico 2 - Porcentagem de equipamentos que emitem radiações ionizantes e não ionizantes seguindo a distribuição na cidade de Ceres-GO.

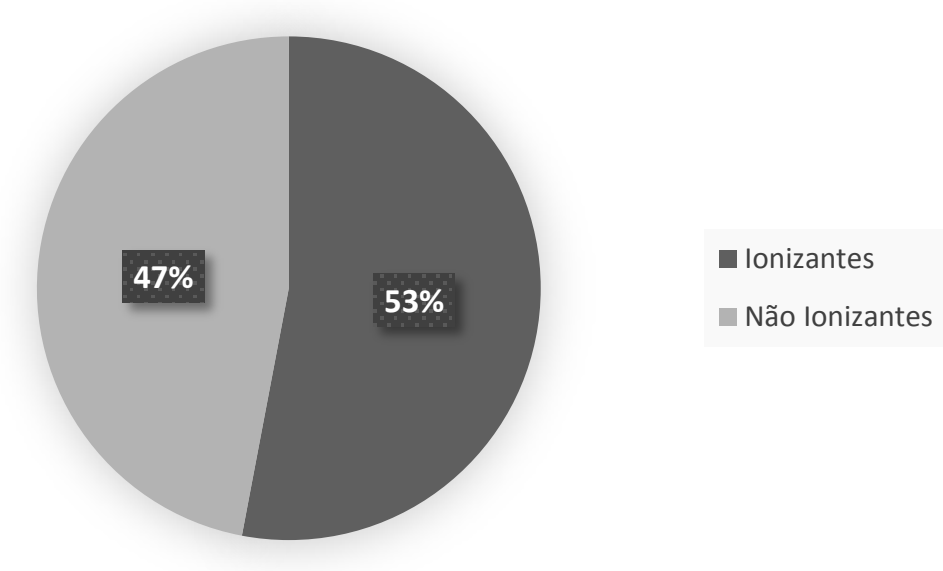

De acordo com o gráfico 2 percebe-se que 53\% dos equipamentos correspondem aos que transmitem radiações ionizantes e $47 \%$ as não ionizantes. Os exames de diagnóstico por imagem são a única fonte de exposição à radiação. Os exames de diagnóstico por imagem que utilizam radiação, normalmente radiografias, como: Angiografia, Tomografia computadorizada, Ressonância magnética, Radiografia simples, Mapeamento por radioisótopos, Ultrassonografia, são uma recursos valiosos no diagnóstico, porém a exposição à radiação apresenta alguns riscos para o paciente em geral são pequenos, porém devem ser levados em consideração. Kay et al (2012) afirmam que a Ressonância Magnética e a Ultrassonografia não utilizam radiação ionizante, e por isso, devem ser preferidos sempre que possível.

Raio-X (radiografia) utilizam uma dose muito pequena de radiação ionizante para produzir as imagens. A Tomografia computadorizada é um dos exames com maior taxa de radiação ionizante. Segundo Tarantino (2017) o Food and Drug Administration (FDA), o órgão do governo americano que regulamenta o setor de saúde, divulga que uma em cada duas mil pessoas tem chance de desenvolver um câncer por conta da superexposição aos raios da tomografia. Para Silva e Marcondes (2018), a radiação ionizante é capaz de ocasionar efeitos crônicos ou agudos na saúde, uma vez que os crônicos são os mais corriqueiros. Estes efeitos podem passar a existir depois de baixas exposições, entretanto no decorrer do tempo, 
pode-se desenvolver de modo lento, na maioria das vezes entre 5 a 30 anos, já a radiação não ionizante não tem energia suficiente para movimentar elétrons dentro de estruturas atômicas.

Paz e Bolognesi (2017) destacam que diferentes exames de diagnóstico requerem diferentes quantidades de radiação, mas a maioria deles usa baixas doses que são, geralmente, consideradas seguras. Por exemplo, a dose de radiação de uma radiografia do tórax é mais que 100 vezes menor que a dose média anual de radiação do ambiente. No entanto, Bacchim Neto (2014) afirmam que se as pessoas fizerem muitos exames de diagnóstico que usam baixas doses ou vários exames que usam altas doses, poderão ser expostas a uma quantidade relativamente grande de radiação. A exposição à radiação é cumulativa, independentemente do intervalo entre os exames. Essa exposição aumenta o risco de câncer e, algumas vezes, causa danos aos tecidos.

O Quadro 3 permite observar que dos 124 equipamentos de diagnóstico por imagem existentes disponíveis nas clínicas de saúde da cidade de Ceres - GO, se encontra na clínica 2 e suas unidades, que possui maior número de equipamentos. A clínica tem a capacidade de oferecer a seus clientes (pacientes) um indicador maior de equipamentos que possam atender a necessidade dos indivíduos, totalizando em 18 equipamentos de diagnósticos prontos para atender a demanda da cidade de Ceres e região. Em seguida a clínica 4 possui um total de 14 equipamentos de diagnósticos e em seguida, a clínica 31 com 11 equipamentos disponíveis para a população.

Quadro 3 - Números de Equipamentos de diagnóstico disponíveis por imagem detalhadamente de cada Clínica de Ceres.

\begin{tabular}{|l|c|c|c|c|c|c|c|}
\hline & DMO & MMG & RM & RX & TC & USG & TOTAL \\
\hline CLÍNICA 1 & 1 & 0 & 0 & 1 & 1 & 2 & $\mathbf{5}$ \\
\hline CLÍNICA 2 & 1 & 2 & 1 & 2 & 1 & 3 & $\mathbf{1 0}$ \\
\hline CLÍNICA 2. Und I & 1 & 3 & 0 & 1 & 0 & 2 & $\mathbf{7}$ \\
\hline CLÍNICA 2. Und III & 0 & 0 & 1 & 0 & 0 & 0 & $\mathbf{1}$ \\
\hline CLÍNICA 3 & 0 & 0 & 0 & 3 & 0 & 2 & $\mathbf{5}$ \\
\hline CLÍNICA 4 & 1 & 3 & 0 & 5 & 1 & 4 & $\mathbf{1 4}$ \\
\hline CLÍNICA 5 & 0 & 0 & 0 & 1 & 0 & 0 & $\mathbf{1}$ \\
\hline CLÍNICA 6 & 0 & 0 & 0 & 2 & 0 & 2 & $\mathbf{4}$ \\
\hline CLÍNICA 7 & 0 & 0 & 0 & 1 & 0 & 1 & $\mathbf{2}$ \\
\hline CLÍNICA 8 & 0 & 0 & 0 & 1 & 0 & 0 & $\mathbf{1}$ \\
\hline CLÍNICA 9 & 0 & 0 & 0 & 0 & 0 & 1 & $\mathbf{1}$ \\
\hline CLÍNICA 10 & 0 & 0 & 0 & 1 & 0 & 0 & $\mathbf{1}$ \\
\hline CLÍNICA 11 & 0 & 0 & 0 & 0 & 0 & 2 & $\mathbf{2}$ \\
\hline
\end{tabular}




\begin{tabular}{|l|c|c|c|c|c|c|c|}
\hline CLÍNICA 12 & 0 & 0 & 0 & 0 & 0 & 1 & $\mathbf{1}$ \\
\hline CLÍNICA 13 & 0 & 0 & 0 & 0 & 0 & 2 & $\mathbf{2}$ \\
\hline CLÍNICA 14 & 0 & 0 & 0 & 1 & 0 & 0 & $\mathbf{1}$ \\
\hline CLÍNICA 15 & 0 & 0 & 0 & 1 & 0 & 0 & $\mathbf{1}$ \\
\hline CLÍNICA 16 & 0 & 0 & 0 & 2 & 1 & 1 & $\mathbf{4}$ \\
\hline CLÍNICA 17 & 0 & 0 & 0 & 2 & 0 & 0 & $\mathbf{2}$ \\
\hline CLÍNICA 18 & 0 & 2 & 0 & 1 & 1 & 3 & $\mathbf{7}$ \\
\hline CLÍNICA 18. DGN & 1 & 1 & 0 & 1 & 1 & 3 & $\mathbf{7}$ \\
\hline CLÍNICA 18. UTI & 0 & 1 & 0 & 1 & 1 & 3 & $\mathbf{6}$ \\
\hline CLÍNICA 19 & 1 & 0 & 0 & 0 & 1 & 3 & $\mathbf{5}$ \\
\hline CLÍNICA 20 & 0 & 0 & 0 & 2 & 0 & 0 & $\mathbf{2}$ \\
\hline CLÍNICA 21 & 0 & 0 & 0 & 1 & 0 & 0 & $\mathbf{1}$ \\
\hline CLÍNICA 22 & 0 & 0 & 0 & 1 & 0 & 1 & $\mathbf{2}$ \\
\hline CLÍNICA 23 & 0 & 0 & 0 & 0 & 0 & 3 & $\mathbf{3}$ \\
\hline CLÍNICA 24 & 0 & 0 & 0 & 0 & 0 & 2 & $\mathbf{2}$ \\
\hline CLÍNICA 25 & 0 & 0 & 0 & 0 & 0 & 1 & $\mathbf{1}$ \\
\hline CLÍNICA 26 & 0 & 1 & 0 & 1 & 1 & 3 & $\mathbf{6}$ \\
\hline CLÍNICA 27 & 0 & 0 & 0 & 2 & 0 & 0 & $\mathbf{2}$ \\
\hline CLÍNICA 28 & 0 & 0 & 0 & 1 & 0 & 0 & $\mathbf{1}$ \\
\hline CLÍNICA 29 & 1 & 1 & 1 & 1 & 1 & 3 & $\mathbf{8}$ \\
\hline CLÍNICA 30 & 0 & 0 & 0 & 0 & 0 & 2 & $\mathbf{2}$ \\
\hline CLÍNICA 31 & 1 & 3 & 0 & 4 & 0 & 3 & $\mathbf{1 1}$ \\
\hline CLÍNICA 32 & 0 & 0 & 0 & 1 & 0 & 0 & $\mathbf{1}$ \\
\hline CLÍNICA 33 & 0 & 0 & 0 & 0 & 0 & 1 & $\mathbf{1}$ \\
\hline CLÍNICA 34 & 0 & 0 & 0 & 0 & 0 & 1 & $\mathbf{1}$ \\
\hline TOTAL & $\mathbf{8}$ & $\mathbf{1 7}$ & $\mathbf{3}$ & $\mathbf{4 1}$ & $\mathbf{1 0}$ & $\mathbf{5 5}$ & $\mathbf{1 2 4}$ \\
\hline
\end{tabular}

$\mathrm{DMO}=$ Densitometria Óssea; $\mathrm{MMG}=$ Mamógrafos; $\mathrm{RM}=$ Ressonância Magnética; $\mathrm{RX}=\mathrm{Raios}-\mathrm{X} ; \mathrm{TC}=$ Tomógrafos Computadorizados USG= Ultrassonografias.

Villar (2015) garante que a evolução de tecnologias aplicadas à medicina diagnóstica auxilia o trabalho dos profissionais e aumenta a segurança dos pacientes quanto ao tratamento de diversos tipos de doenças. No entanto, não adianta uma clínica ter vários tipos de equipamentos de diagnósticos de imagem e não oferecer qualidade em seus exames. Assim, Silva (2012) ressaltam que para que o serviço permaneça proporcionando diagnóstico de boa qualidade, sustentando a qualidade da imagem, tornando cada dia mais otimização as doses e com treinamento apropriado dos técnicos, precisa-se ser implementado Programas de Garantia da Qualidade (PGQ). Um programa de garantia de qualidade monitora o desempenho destes equipamentos, materiais e demais procedimentos. 
Venâncio et al (2014) relatam que no Brasil os programas de qualidade em imagem são elaborados e aplicados através de portarias, essas por sua vez compostas por leis e decretos. O Ministério da saúde (2012) divulga que as portarias do Ministério da Saúde número 453 de $1^{\circ}$ de junho de 2008 e a portaria número 531 de 26 de março de 2012 são responsáveis por estabelecer as normas a serem cumpridas a fim de garantir a qualidade da imagem. Essas portarias estabelecem o regulamento exclusivamente de imagens adquiridas pelo uso dos raios $\mathrm{X}$.

\section{CONCLUSÃO}

Os equipamentos de exames de diagnósticos existentes na cidade de Ceres-GO, são satisfatórios para atender toda a população da cidade e região. Os exames de diagnósticos são de suma importância para auxiliar o médico na tomada de decisão para que se possa dar o diagnóstico correto visando o tratamento saúde/doença.

Contudo foi possível verificar a distribuição dos equipamentos de diagnóstico por imagem disponíveis na cidade de Ceres - GO, as informações do sistema de saúde disponíveis na rede mundial de computadores, realizada pelo Datasus, contribuem para a melhoria do sistema público de saúde, na medida em que possibilitam que os dados sejam tabelados e analisados de forma a enfocar uma determinada área da saúde. Além disso, estudos como este podem servir de base para futuros trabalhos na área de radiologia diagnóstica

Por fim, conclui-se que embora o processo de cadastramento de informações no banco de dados do CNES/ Datasus ainda não tenha alcançado sua última etapa, a certificação e os dados disponíveis relativos ao município de Ceres -GO já permitem uma análise bastante realista do dos equipamentos de diagnóstico por imagem instalado na cidade.

\section{REFERÊNCIAS}

ANDRADE, Z.T.M. Da efetivação do direito à saúde no Brasil. In: Âmbito Jurídico, Rio Grande, XIV, n. 86, mar 2017. Disponível em:

http://www.ambitojuridico.com.br/site/?n_link=revista_artigos_leitura\&artigo_id=9037\&revi sta_caderno=9. Acesso em 07/01/2019.

ARAÚJO, P.N.B et al. Mapeamento dos equipamentos e exames de diagnóstico por imagem no estado de São Paulo. Tekhne e Logos, Botucatu, SP, v.7, n.1, Abril, 2016. 
BACCHIM NETO, F.A. Avaliação da Exposição à Radiação no Médico Intervencionista em Procedimentos no Setor de Hemodinâmica. IBB/UNESP - Instituto de Biociências, 2014. Disponível em https://repositorio.unesp.br/handle/11449/145383. Acesso em 16/01/2019.

BENEVIDES, C. No SUS e na rede privada, desigualdade no acesso a aparelhos de diagnóstico. 2014. Disponível em https://oglobo.globo.com/brasil/no-sus-na-rede-privadadesigualdade-no-acesso-aparelhos-de-diagnostico-12121995. Acesso em 12/01/2019.

BONETTI, C.I.et al. Estudo da interferência de medicamentos em exames laboratoriais. 6 Simpósio de Ciências Farmacêuticas do Mercosul, X CISDEM - Fórum Internacional, 16 a 18 novembro, Cascavel, Paraná, 2016.

BRASIL, MINISTÉRIO DA SAÚDE. Portaria N 531, De 26 de Março De 2012. 2012. Disponível em http://bvsms.saude.gov.br/bvs/saudelegis/gm/2012/prt0531_26_03_2012.html. Acesso em 16/01/2019.

BUSHONG, S.C. Ciências radiológicos para tecnólogos: física, biologia e proteção. Rio de Janeiro: Elsevier, 2010.

CADASTRO NACIONAL DE ESTABELECIMENTOS DE SAUDE (CNES). Disponível em:http://cnes.datasus.gov.br/pages/estabelecimentos/consulta.jsp?earch=CERES\&fbclid=Iw AR2LIEK5iDKOHZ03acUk2QavZ21j53qLGWx-T_xTeSrt805Nb0gg8wnr56c> Acesso em: $17 / 11 / 2018$.

CAMILO, A.V et al. A Deformidade de Crânio plagiocefalia. Revista de Medicina e Saúde de Brasília, nº, (Supl.1), 2016.

CAMPANA, G.A et al. Tendências em medicina laboratorial. J. Bras. Patol. Med. Lab., Rio de Janeiro, v. 47, n. 4, p. 399-408, Aug. 2011.

CARVALHO. C. Radiologia digital. 2017. Disponível em files.sindiradiologia.org.br/200000347-63913648a5/RADIOLOGIA\%20DIGITAL.ppt. Acesso em 08/01/2019. 
COUTO FILHO, A.F. A realidade da saúde no Brasil. Boletim Jurídico, Uberaba/MG, a. 4, no 190. 2016. Disponível em:

http://www.boletimjuridico.com.br/doutrina/texto.asp?id=1466> Acesso em: 06/01/2019.

ECHER, T.L. Medicamentos interferentes em exames laboratoriais. 2013. Disponível em https://repositorio.ucb.br/jspui/handle/123456789/6793. Acesso em 05/01/2019.

GASPARIN, D. Efeitos biológicos da radiação ionizantes. 2013. Disponível em http://tcconline.utp.br/wp-content/uploads/2013/08/efeitos-biologicos-da-radiacao ionizante.pdf. Acesso em 08/01/2019.

INSTITUTO BRASILEIRO DE GEOGRAFIA E ESTATISTICA (IBGE). População no último censo. 2018. Disponível em :<https://cidades.ibge.gov.br/brasil/go/ceres/panorama>. Acesso em 20/11/2018.

KAY, F.U et al. Diretrizes Assistenciais: Radiação Ionizante nos Estudos Radiológicos. Versão eletrônica atualizada em Julho - 2012. Comitê sobre Radiação Ionizante (ComRadI). 2012. Disponível em http://www.saudedireta.com.br/docsupload/1740529943radiacaoionizanteestudos_radiologics.pdf. Acesso em 14/01/2019.

LEYTON, F et al. Riscos da Radiação X e a Importância da Proteção Radiológica na Cardiologia Intervencionista: Uma Revisão Sistemática. Rev Bras Cardiol Invasiva, vol.22, $\mathrm{n}^{\mathrm{o}} .1, \mathrm{p} .: 87-98,2014$.

MADRIGANO, R.R et al. Avaliação do conhecimento de médicos não radiologistas sobre aspectos relacionados à radiação ionizante em exames de imagem. Radiol Bras, Jul/Ago; vol. 47, no.4, p.:210-216, 2014.

MONTEIRO, D.N.B. Estudo sobre a Visualização de Imagens Médicas Obtida por Exames. Virtuais. Dissertação de Mestrado submetida ao Programa de Pós-Graduação em Computação da Universidade Federal Fluminense, 2015. Disponível em http://www2.ic.uff.br/PosGraduacao/Dissertacoes/279.pdf. Acesso em 10/01/10'9.

NASCIMENTO K. N. F.; NASCIMENTO K. N. F. Mapeamento participativo: mapa dos equipamentos de saúde do município de Conde/PB. In: Simpósio Brasileiro de Ciências Geodésicas e Tecnologias da Geoinformação, 4., 2012, Recife. Anais... Recife: Universidade Federal de Pernambuco, 2012. p.1-5. 
NASCIMENTO, F. B et al. Análise dos principais métodos de diagnóstico de câncer de mama como propulsores no processo inovativo. Arq Med, Porto, v. 29, n. 6, p. 153-159, dez. 2015.

OKUNO, E. Efeitos biológicos das radiações ionizantes. Acidente radiológico de Goiânia. Rev. Est. Avan., São Paulo, v. 27, n. 77, set. 2013.

PAZ, A; BOLOGNESI, L. Radioproteção aplicada ao serviço de hemodinâmica. Tekhne e Logos, Botucatu, SP, v.8, n.1, abril, 2017.

SILVA, H.P et al. Caxias, M.C.L. Benefícios para alguns, prejuízos para muitos: razões e implicações da adoção da dupla porta de entrada em hospitais universitários. Saúde soc., vol.25, n.3, pp.808-820, 2016.

SILVA, Luiz Cláudio Nunes da. Vigilância da Qualidade em Mamografia no Estado do Paraná: uma análise de viabilidade. 2012. Disponível em http://gsp.cursoscad.ufsc.br/wp/wp-content/uploads/2013/03/Anais-GSP-Volume-5-Artigo11.pdf. Acesso em 17/01/2019.

SILVA, P.P; MARCONDES, A.L. Otimização da dose de radiação ionizante em tomografia computadorizada. Tekhne e Logos, Botucatu, SP, v.9, n.1, abril, 2018.

SILVA, W.R. Sistemas de imagens digitais com aplicabilidade na odontologia. 2 Monografia apresentada a Faculdade de Odontologia da Universidade Federal do Rio Grande do Sul, ao curso de especialização em Radiologia Odontológica e Imaginologia. 2011. Disponível em https://lume.ufrgs.br/handle/10183/28710. Acesso em 02/01/2019.

SOUZA, A.M.V et al. Solicitação de exames diagnósticos no contexto da Atenção Primária à Saúde: uma revisão integrativa. Renome. Revista norte mineira de enfermagem. 2014. Disponível em http://www.renome.unimontes.br/index.php/renome/article/view/82/104. Acesso em 19/11/2018.

TARANTINO, M. O perigo invisível da tomografia. 2017. Disponível em https://istoe.com.br/286_O+perigo+invisivel+da+tomografia/. Acesso em 15/01/2019.

VENÂNCIO, R.B et al. Medidas para implantação programa de qualidade de imagem em mamografia. 2014. Disponível em http://www.canal6.com.br/cbeb/2014/artigos/cbeb2014_submission_262.pdf. Acesso em 16 /01/2019. 
VILLAR, Vanessa Cristina Felippe Lopes et al. A evolução da qualidade da imagem em mamografia no Estado do Rio de Janeiro. 2015. Disponível em

http://www.rb.org.br/detalhe_artigo.asp?id=2611\&idioma=Portugues. Acesso em 16/01/2019. 